Pacific

Journal of

Mathematics

BASS NUMBERS OF SEMIGROUP-GRADED LOCAL COHOMOLOGY

David Helm and Ezra Miller 


\title{
BASS NUMBERS OF SEMIGROUP-GRADED LOCAL COHOMOLOGY
}

\author{
DAVID HELM AND EzRA Miller
}

\begin{abstract}
Given a module $M$ over a ring $R$ that has a grading by a semigroup $Q$, we present a spectral sequence that computes the local cohomology $H_{I}^{i}(M)$ at any graded ideal $I$ in terms of Ext modules. We use this method to obtain finiteness results for the local cohomology of graded modules over semigroup rings. In particular we prove that for a semigroup $Q$ whose saturation $Q^{\text {sat }}$ is simplicial, and a finitely generated module $M$ over $k[Q]$ that is graded by $Q^{\mathrm{gp}}$, the Bass numbers of $H_{I}^{i}(M)$ are finite for any $Q$-graded ideal $I$ of $k[Q]$. Conversely, if $Q^{\text {sat }}$ is not simplicial, we find a graded ideal $I$ and graded $k[Q]$-module $M$ such that the local cohomology module $H_{I}^{i}(M)$ has infinite-dimensional socle. We introduce and exploit the combinatorially defined essential set of a semigroup.
\end{abstract}

\section{Introduction.}

The local cohomology modules $H_{I}^{i}(M)$ for finitely generated modules $M$ over noetherian rings $R$ have been studied for several decades. When $I$ is a maximal ideal of $R$ the local cohomology of $M$ is fairly well-understood (and reasonably well-behaved), but for general ideals $I$ much less is known, and the behavior can be quite bad. For instance, Hartshorne [Har70] has shown that the Bass numbers of $H_{I}^{i}(M)$ need not be finite for general $I$ and $R$.

Recently, however, some progress has been made in special cases. When $R$ is a regular local ring containing a field, Lyubeznik [Lyu93], and Huneke and Sharp [HS93] have shown that $H_{I}^{i}(M)$ has finite Bass numbers. In the same spirit (albeit by different techniques), Yanagawa [Yan01] has shown that if $\omega$ is the canonical module of a simplicial and normal semigroup ring and $I$ is a monomial ideal, then $H_{I}^{i}(\omega)$ has finite Bass numbers.

Our approach is a substantial generalization of that found in [Yan01]. We consider a noetherian ring $R$ graded by a semigroup $Q$, and modules over $R$ graded by $Q^{\text {gp }}$. In this setting, we introduce a functor called the $\check{C}$ ech hull (Section 2), which allows us to recover the full local cohomology of a finitely generated module $M$ from the portion of the local cohomology that lies in those graded degrees that are elements of $Q$ (Section 3). This piece of the 
local cohomology is often easier to understand than the entire module; in particular it is finitely generated when $R=k[Q]$ for an affine semigroup $Q$ (see Proposition 4.3, or Corollary 5.3 along with Proposition 5.4). Using this fact we prove several finiteness results for the local cohomology of $Q^{\mathrm{gp}}$ graded modules over affine semigroup rings (Section 5). In particular we show (without Cohen-Macaulay hypotheses) that when the saturation $Q^{\text {sat }}$ is simplicial, $H_{I}^{i}(M)$ has finite Bass numbers.

The converse holds, as well: Our construction of a local cohomology module with infinite-dimensional socle when $Q^{\text {sat }}$ is not simplicial (Section 7) contains Hartshorne's counterexample to Grothendieck's conjecture [Har70] as the simplest special case. The constructions are polyhedral in nature, exploiting a new combinatorial structure, the essential set of a semigroup (Section 6). Properties of the essential set govern the associated primes, and to some extent the module structure, of the local cohomology of the canonical module.

The reader interested in affine semigroup rings rather than general semigroup gradings need not endure anything in Sections 2-4 except Proposition 4.1 (which can be taken as a definition) and the two paragraphs preceding it. Instead, begin with Section 7 and the first half of Section 6 (through Example 6.5) - in either order - noting especially Theorem 7.1, which contains our main results on local cohomology over affine semigroup rings. Then continue with Proposition 4.1 and Section 5. The required results from other parts of the paper can be referred to as necessary.

\section{The Čech hull.}

Let $Q$ be a cancellative, commutative semigroup, and $Q^{\text {gp }}$ its Grothendieck group, i.e., the group obtained from $Q$ by adjoining an inverse for every element. (The reader may safely assume for the purposes of this paper that $Q$ is affine - that is, a finitely generated submonoid of $\mathbb{Z}^{d}$ with $Q^{\text {gp }}=\mathbb{Z}^{d}$; indeed, we will make this assumption starting in Section 4. However, we hope that the extra generality in this section and the next will be useful for more general semigroup gradings, such as those arising in the Cox homogeneous coordinate rings of toric geometry [Cox95].) We say a ring $R$ is $Q$-graded and an $R$-module $M$ is $Q^{\text {gp }}$-graded if we are given direct sum decompositions

$$
R=\bigoplus_{a \in Q} R_{a} \quad \text { and } \quad M=\bigoplus_{\alpha \in Q^{\mathrm{gp}}} M_{\alpha}
$$

such that $R_{a} R_{b} \subseteq R_{a+b}$ and $R_{a} M_{\beta} \subseteq M_{a+\beta}$. The category of $Q^{\text {gP }}$-graded modules is henceforth denoted by $\mathcal{M}$.

A morphism $M \rightarrow N$ in $\mathcal{M}$ is a degree-preserving $R$-module homomorphism; i.e., a map $f$ of $R$-modules such that $f\left(M_{\alpha}\right) \subseteq N_{\alpha}$. We denote by $\operatorname{Hom}_{R}(M, N)$ the $R_{0}$-module of such morphisms and by $\underline{\operatorname{Hom}}_{R}(M, N)$ the 
$Q^{\text {gp }}$-graded $R$-module

$$
\underline{\operatorname{Hom}}_{R}(M, N)=\bigoplus_{\alpha \in Q^{\mathrm{gp}}} \operatorname{Hom}_{R}(M, N(\alpha))=\bigoplus_{\alpha \in Q^{\mathrm{gp}}} \operatorname{Hom}_{R}(M(-\alpha), N) .
$$

Here, the $Q^{\mathrm{gp}}$-graded $R$-module $N(\alpha)$ is the shift of $N$ by $\alpha$, defined by $N(\alpha)_{\beta}=N_{\alpha+\beta}$.

For a subset $S \subseteq Q^{\text {gp }}$ closed under the action of $Q$, we define the $S$-graded part $M_{S} \subseteq M$ to be

$$
M_{S}=\bigoplus_{\alpha \in S} M_{\alpha}
$$

We frequently consider the case in which $S=\alpha+Q$ for some $\alpha \in Q^{\mathrm{gp}}$. In particular, taking $M_{Q}$ yields the part of $M$ graded by elements of $Q$. Taking $S$-graded parts is functorial and exact, for any $S$.

The theory we develop below revolves around the following question: To what extent can we recover a module $M$ from its $Q$-graded part $M_{Q}$ ? If $M$ is finitely generated, for example, then although we may not be able to get $M$ from $M_{Q}$, we can shift by some $a \in Q$ to get $M(-a)=M(-a)_{Q}$. Therefore, the question is more meaningful for infinitely generated modules, such as the local cohomology modules of a finitely generated module. We will find that these belong to a certain class of modules that can be recovered from $Q$-graded parts of other modules by a functor $\check{C}$ that we call the $\check{C}$ ech hull, as defined by the next result.

Theorem 2.1. The functor $(-)_{Q}: \mathcal{M} \rightarrow \mathcal{M}$ taking $Q$-graded parts has a right adjoint $\check{C}$; that is, there exists a functor $\check{C}$ and natural isomorphisms

$$
\operatorname{Hom}_{R}\left(M_{Q}, N\right)=\operatorname{Hom}_{R}(M, \check{C} N)
$$

for any $M, N \in \mathcal{M}$.

Proof. Given $N$, we explicitly construct $\check{C} N$ by defining

$$
\begin{aligned}
(\check{C} N)_{\alpha} & =\operatorname{Hom}_{R}\left(R_{Q-\alpha}, N(\alpha)\right) \\
& =\operatorname{Hom}_{R}\left(R_{Q-\alpha}(-\alpha), N\right) .
\end{aligned}
$$

The multiplication maps

$$
R_{b} \otimes_{R_{\mathbf{0}}}(\check{C} N)_{\alpha} \rightarrow(\check{C} N)_{b+\alpha}
$$

are given by taking $r \otimes \phi$ to $(x \mapsto \phi(r x))$. This is well-defined since multiplication by $r \in R_{b}$ is a degree zero map $R_{Q-\alpha-b}(-\alpha-b) \rightarrow R_{Q-\alpha}(-\alpha)$.

Note that if $a \in Q$ then $R_{Q-a}=R$, so

$$
(\check{C} N)_{a}=\operatorname{Hom}_{R}\left(R_{Q-a}(-a), N\right)=\operatorname{Hom}_{R}(R(-a), N)=N_{a},
$$

whence $(\check{C} N)_{Q}=N_{Q}$. Therefore, given an element of $\operatorname{Hom}_{R}(M, \check{C} N)$, taking its $Q$-graded part gives an element of $\operatorname{Hom}_{R}\left(M_{Q}, N_{Q}\right)$. This last module is $\operatorname{Hom}_{R}\left(M_{Q}, N\right)$ (since degree zero maps from $M_{Q}$ to $N$ must land in $N_{Q}$ ), so we have produced a natural $\operatorname{map} \operatorname{Hom}_{R}(M, \check{C} N) \rightarrow \operatorname{Hom}_{R}\left(M_{Q}, N\right)$. 
Conversely, if $f \in \operatorname{Hom}_{R}\left(M_{Q}, N\right)$ then for each $\alpha \in Q^{\text {gp }}$ we have a map

$$
M_{\alpha} \rightarrow(\check{C} N)_{\alpha}=\operatorname{Hom}_{R}\left(R_{Q-\alpha}, N(\alpha)\right) \text { defined by } \quad x \mapsto(r \mapsto f(r x)) \text {. }
$$

This is well-defined since if $r \in R_{Q-\alpha}$ and $x \in M_{\alpha}$, then $r x \in M_{Q}$, so we can evaluate $f(r x)$. We thus obtain a well-defined element of $\operatorname{Hom}_{R}(M, \check{C} N)$, whose $Q$-graded part is just $f$. This gives the natural inverse map for our bijection.

Remark 2.2. It is clear by looking at the graded pieces of $\check{C}$ that it is left exact but not right exact, and that its derived functors are given in terms of Ext modules; that is $\left(\left(R^{i} \check{C}\right) M\right)_{\alpha}=\operatorname{Ext}_{R}^{i}\left(R_{Q-\alpha}(-\alpha), M\right)$. Since $R_{Q-\alpha}(-\alpha)$ is supported on $Q \subset Q^{\text {gp }}$, both the Cech hull and its derived functors depend only on the $Q$-graded part of $M$.

Remark 2.3. The Čech hull was defined in [Mil00] for polynomial rings. In this case $Q=\mathbb{N}^{d}, R=k[Q]$, and $\operatorname{Hom}_{R}\left(R_{Q-\alpha}(-\alpha), M\right)=M_{\alpha^{+}}$, where $\alpha^{+}$is obtained from $\alpha$ by zeroing out the negative coordinates. Thus our definition of $\check{C}$ agrees with the one in [Mil00]. Note that the Cech hull is exact in this case, since $R_{Q-\alpha}$ is free for all $\alpha$, so the Ext modules that make up the graded pieces of $R^{i} \check{C}$ vanish.

Example 2.4. Let $Q \subset \mathbb{Z}^{2}$ be the semigroup generated by $(0,2),(1,1)$, and $(2,0)$, so that $Q^{\text {gp }} \subset \mathbb{Z}^{2}$ is a lattice of index 2 . Take $R=k[Q]$, graded by $Q$. If $\alpha=(x, y) \in Q^{\mathrm{gp}}$, then $x$ and $y$ have the same parity. If $x$ and $y$ are even, or if $x$ and $y$ have the same sign, then $R_{Q-\alpha}(-\alpha)$ is free. On the other hand, if $x$ is odd and negative while $y$ is odd and positive, then $R_{Q-\alpha}(-\alpha)$ is generated in degrees $(1, y)$ and $(0, y+1)$. Moreover, one has an exact sequence:

$0 \rightarrow R_{Q-\alpha}(-\alpha-(1,1)) \longrightarrow R(-1,-y) \oplus R(0,-y-1) \longrightarrow R_{Q-\alpha}(-\alpha) \rightarrow 0$.

Splicing homological and graded shifts of this short exact sequence together gives a free resolution $F$. of $R_{Q-\alpha}(-\alpha)$ such that $F_{i}=R(-1-i,-y-i) \oplus$ $R(-i,-y-i-1)$. Since $Q$ is symmetric in $x$ and $y$, a similar result holds with $x$ and $y$ reversed.

We now have free resolutions of $R_{Q-\alpha}(-\alpha)$ for every $\alpha \in Q^{\mathrm{gp}}$, and we can use them to compute the Cech hull and its derived functors. For instance, consider the module $k(-u,-v)$ consisting of a single copy of the residue field $k$, supported in a degree $(u, v)$ satisfying $v>u>1$. Then for $\alpha=(x, y)$, we find that $\operatorname{Hom}_{R}\left(R_{Q-\alpha}(-\alpha), k(-u,-v)\right)$ is only nontrivial if $\alpha=(u, v)$. Since Equation (1) implies that

$$
R^{i} \check{C}(k(-u,-v))_{\alpha} \cong \operatorname{Ext}_{R}^{i}\left(R_{Q-\alpha}(-\alpha), k(-u,-v)\right),
$$

it follows that $\check{C}(k(-u,-v))=k(-u,-v)$. In contrast, $R^{i} \check{C}(k(-u,-v))$ for $i>1$ can only be nonzero in degrees $\alpha$ for which $x$ and $y$ are odd and of differing sign, since $R_{Q-\alpha}(-\alpha)$ is free otherwise. 
Suppose we have such an $\alpha$, and let $F$. be the corresponding free resolution of $\alpha$ constructed above. Then $\operatorname{Hom}_{R}\left(F_{i}, k(-u,-v)\right.$ ) is nonzero if (and only if) one of the generators of $F_{i}$ sits in degree $(u, v)$. Referring to the expression for the degrees of the $F_{i}$ in terms of $\alpha$ (and remembering that we assumed $v>u>1)$, we find that $\operatorname{Hom}_{R}\left(F_{i}, k(-u,-v)\right)$ is nonzero if and only if $i=u$ and $y=v-u-1$ or if $i=u-1$ and $y=v-u+1$. In other words, $R^{i} \check{C}(k(-u,-v))$ vanishes except when $i \in\{u-1, u\}$. Moreover, $R^{u-1} \check{C}(k(-u,-v))$ is supported in those degrees $\alpha$ such that $x$ is odd and negative and $y=v-u+1$, while $R^{u} \check{C}(k(-u,-v))$ is supported in those degrees $\alpha$ such that $x$ is odd and negative and $y=v-u-1$.

To summarize, when $v>u>1$, we have:

- $\check{C}(k(-u,-v))=k(-u,-v)$

- $R^{u-1} \check{C}(k(-u,-v))=k\left[\mathbf{x}^{-(2,0)}\right](-1,-v+u-1)$

- $R^{u} \check{C}(k(-u,-v))=k\left[\mathbf{x}^{-(2,0)}\right](-1,-v+u+1)$

and all other derived functors of $\check{C}$ vanish. Here, $\mathbf{x}^{\alpha} \in k\left[Q^{\mathrm{gp}}\right]$ is the element corresponding to $\alpha \in Q^{\text {gp }}$.

\section{Local cohomology.}

In this section we study the interaction of the Cech hull with the functor $\Gamma_{I}$, which takes a module $M$ to the submodule annhilated elementwise by some power of the ideal $I$. If $I$ is graded, then $\Gamma_{I}$ takes the category $\mathcal{M}$ of $Q^{\mathrm{gP}}$-graded modules to itself.

Proposition 3.1. If $R$ is noetherian, then $\check{C}$ and $\Gamma_{I}$ commute: $\Gamma_{I} \check{C}=$ $\check{C} \Gamma_{I}$.

Proof.

$$
\begin{aligned}
\left(\Gamma_{I} \check{C} M\right)_{\alpha} & =\bigcup_{n}\left\{x \in \operatorname{Hom}_{R}\left(R_{Q-\alpha}(-\alpha), M\right) \mid I^{n} x=0\right\} \\
& =\operatorname{Hom}_{R}\left(R_{Q-\alpha}(-\alpha), \Gamma_{I} M\right) \\
& =\left(\check{C} \Gamma_{I} M\right)_{\alpha} .
\end{aligned}
$$

Henceforth we assume $R$ is noetherian. In Proposition 3.3 we shall apply the spectral sequence of a composite functor to the functors $\Gamma_{I} \check{C}$ and $\check{C} \Gamma_{I}$. In order to do this we use the fact that both $\Gamma_{I}$ and $\check{C}$ take injectives to injectives. For $\Gamma_{I}$ this is standard; for $\check{C}$ this follows from Lemma 3.2, whose extra precision is vital for Section 5 .

Lemma 3.2. Let $J$ be an indecomposable injective in $\mathcal{M}$. Then $\check{C} J=0$ if $J_{Q}=0$, and $\check{C} J=J$ otherwise. In particular, $\check{C}$ takes injectives to injectives. 
Proof. The first statement is clear, since $\check{C}$ depends only on the $Q$-graded portion of a module. The last statement follows from the others, using the fact that every injective is a direct sum of indecomposable injectives (this uses the noetherian hypothesis).

For the remaining statement, write $J=\underline{E}(R / \mathfrak{p})(\alpha)$ for some prime $\mathfrak{p}$ of $R$ and $\alpha \in Q^{\mathrm{gp}}$. Then $(R / \mathfrak{p})(\alpha)$ is an essential submodule of $J$, so since $J_{Q}$ is nonzero, $(R / \mathfrak{p})(\alpha)_{Q}$ is nonzero. Now $(R / \mathfrak{p})(\alpha)_{Q}$ is an essential submodule of $(R / \mathfrak{p})(\alpha)$ (since $R / \mathfrak{p}$ is an integral domain), so it is an essential submodule of $J$. Thus in particular $J_{Q}$ is an essential submodule of $J$.

The inclusion $J_{Q} \rightarrow J$ induces a map $\phi: J \rightarrow \check{C} J$, by the adjointness property of $\check{C}$. Moreover, $\phi$ is injective, since it restricts to the identity on the essential submodule $J_{Q}$. Thus $J$ is a direct summand of $\check{C} J$, since $J$ is injective. We claim that $\check{C} J$ is an essential extension of $J$, from which the result follows immediately.

Let $x \in(\check{C} J)_{\alpha}=\operatorname{Hom}_{R}\left(R_{Q-\alpha}(-\alpha), J_{Q}\right)$ be a nonzero homogeneous element. Then there is an $r \in R$ such that $x(r)$ is nonzero, and then $r x=x(r)$ is a nonzero element of $J_{Q}$. Thus $\breve{C} J$ is an essential extension of $J_{Q}$ and hence of $J$ as well.

Proposition 3.3. Let $M$ be a graded $R$-module. There are spectral sequences $E(M)$ and $F(M)$ described by

$$
\begin{aligned}
& E_{2}^{p, q}(M)=R^{p} \check{C} H_{I}^{q}(M) \Rightarrow R^{p+q}\left(\Gamma_{I} \check{C}\right) M \\
& F_{2}^{p, q}(M)=H_{I}^{p}\left(R^{q} \check{C} M\right) \Rightarrow R^{p+q}\left(\Gamma_{I} \check{C}\right) M .
\end{aligned}
$$

Proof. These are spectral sequences for the composite functors $\check{C} \Gamma_{I}$ and $\Gamma_{I} \check{C}$ [Wei94], using Proposition 3.1 along with Lemma 3.2 and the fact that $\Gamma_{I}$ takes injectives to injectives.

Example 3.4. We return to the case of Remark 2.3; that is, $R=k\left[\mathbb{N}^{d}\right]$. Here $\check{C}$ is exact, and so the spectral sequences $E(M)$ and $F(M)$ both collapse. The proposition simply says that $H_{I}^{i}(\check{C} M)=\check{C} H_{I}^{i}(M)$. The right derived functors of the Čech hull measure the degree to which this equality fails in other rings.

Ultimately, the goal of this section is Theorem 3.10, which describes how the local cohomology modules of $M$ can be reconstructed from a finite collection of submodules thereof, using the Čech hull and its derived functors. After a choosing a certain $\alpha \in Q^{\text {gp }}$, this is accomplished by a spectral sequence $E(M(-\alpha))$ that only depends on the (finitely generated) $Q$-graded parts of the local cohomology modules of $M(-\alpha)$, since $R^{p} \check{C} H_{I}^{q}(-)$ depends only on the $Q$-graded part of $H_{I}^{q}(-)$.

For this approach to work, we of course need $R^{p+q}\left(\Gamma_{I} \check{C}\right) M$ to be a local cohomology module. To this end, we use the spectral sequence $F(M)$. 
Although the filtration that arises from $F(M)$ is generally nontrivial, we avoid this nuisance by replacing $M$ with a suitable $Q^{\mathrm{gp}}$-graded shift, forcing $F$ to collapse in low cohomological degree. We find this suitable shift in Corollary 3.6 using Proposition 3.5, which is interesting in its own right.

Proposition 3.5. Let $J \cdot$ be a minimal injective resolution of a finitely generated module $M \in \mathcal{M}$. Let $\mathfrak{p}$ be a homogeneous prime of $R$, let $\mathfrak{m}$ be a homogeneous maximal ideal containing $\mathfrak{p}$, and let $c=\operatorname{dim}(R / \mathfrak{p})-\operatorname{dim}(R / \mathfrak{m})$. If every indecomposable summand of $\Gamma_{\mathfrak{m}} J^{i+c}$ has nonzero $Q$-graded part, then every indecomposable summand of $J^{i}$ isomorphic to a shift of $\underline{E}(R / \mathfrak{p})$ has nonzero Q-graded part.

Proof. Inverting all homogeneous elements outside $\mathfrak{m}$ fixes all shifts of $\underline{E}(R / \mathfrak{p})$ as well as $\Gamma_{\mathfrak{m}} J^{i}$, so we assume henceforth that $\mathfrak{m}$ is the unique maximal homogeneous ideal of $R$.

We begin with the case $c=1$. Using $(-)_{(\mathfrak{p})}$ to denote the localization by all homogeneous elements outside of $\mathfrak{p}$, it is a standard fact (in [BH93, p. 101], for instance) that $\left(\underline{\operatorname{Ext}}_{R}^{i}(R / \mathfrak{p}, M)\right)_{(\mathfrak{p})}=\left(\underline{\operatorname{Hom}}_{R}\left(R / \mathfrak{p}, J^{i}\right)\right)_{(\mathfrak{p})} \subset$ $\left(\Gamma_{\mathfrak{p}} J^{i}\right)_{(\mathfrak{p})}$ is an essential extension. Therefore, we need only show that every indecomposable submodule of the free $(R / \mathfrak{p})_{(\mathfrak{p})}$-module $\left(\underline{\operatorname{Ext}}_{R}^{i}(R / \mathfrak{p}, M)\right)_{(\mathfrak{p})}$ has nonzero $Q$-graded part.

Choose a homogeneous element $x \in \mathfrak{m} \backslash \mathfrak{p}$, and define $L$ by the exact sequence

$$
0 \rightarrow R / \mathfrak{p} \stackrel{x}{\longrightarrow} R / \mathfrak{p} \longrightarrow L \rightarrow 0,
$$

where $\stackrel{x}{\rightarrow}$ is multiplication by $x$. The long exact sequence for $\underline{\operatorname{Ext}}_{R}(-, M)$ provides a right exact sequence

$$
\underline{\operatorname{Ext}}_{R}^{i}(R / \mathfrak{p}, M) \stackrel{x}{\longrightarrow} \underline{\operatorname{Ext}}_{R}^{i}(R / \mathfrak{p}, M) \longrightarrow L(i, x, M) \rightarrow 0
$$

for the appropriate submodule $L(i, x, M) \subseteq \underline{\operatorname{Ext}}_{R}^{i+1}(L, M)$. Tensoring with $R / \mathfrak{m}$ yields an isomorphism $R / \mathfrak{m} \otimes \underline{\operatorname{Ext}}_{R}^{i}(R / \mathfrak{p}, M) \cong R / \mathfrak{m} \otimes L(i, x, M)$ since multiplication by $x$ becomes the zero map. Nakayama's lemma implies that $\underline{\operatorname{Ext}}_{R}^{i}(R / \mathfrak{p}, M)$, and hence $\left(\underline{\operatorname{Ext}}_{R}^{i}(R / \mathfrak{p}, M)\right)_{(\mathfrak{p})}$, is generated by elements in degrees $\gamma \in Q^{\mathrm{gp}}$ such that $L(i, x, M)_{\gamma} \subseteq \underline{\operatorname{Ext}}_{R}^{i+1}(L, M)_{\gamma} \neq 0$.

Now $\operatorname{Ext}_{R}^{i+1}(L, M)$ is the $(i+1)^{\text {st }}$ cohomology of the complex $\underline{\operatorname{Hom}}_{R}\left(L, J^{\bullet}\right)$ $\subset \Gamma_{\mathfrak{m}} J^{\bullet}$, whose socle subcomplex $\underline{\operatorname{Hom}}_{R}\left(R / \mathfrak{m}, J^{\bullet}\right) \subseteq \underline{\operatorname{Hom}}_{R}\left(L, J^{\bullet}\right)$ (the inclusion being induced by the surjection $L \rightarrow R / \mathfrak{m})$ is equal to $\operatorname{Ext}_{R}(R / \mathfrak{m}, M)$. The hypothesis on $\left(\Gamma_{\mathfrak{m}} J^{i}\right)_{Q}$ in the Proposition implies that $\underline{\operatorname{Ext}}_{R}^{i+1}(R / \mathfrak{m}, M)$ must in fact equal its $Q$-graded part. Given any degree $\gamma \in Q^{\text {gp }}$ for which $\underline{\operatorname{Ext}}_{R}^{i+1}(L, M)_{\gamma} \neq 0$, we therefore can find a homogeneous element $r \in R$ such that $\gamma+\operatorname{deg}(r) \in Q$. Note that $\mathfrak{p}$ annihilates $L$ and hence also $\underline{\operatorname{Hom}}_{R}\left(L, J^{\bullet}\right)$, so we can always find our element $r$ outside of $\mathfrak{p}$. When we invert $r$ to form 
the localization $\left(\underline{\operatorname{Ext}}_{R}^{i}(R / \mathfrak{p}, M)\right)_{(\mathfrak{p})}$, any generator $y$ in degree $\gamma$ can be replaced by the generator $r y$ whose degree is in $Q$. This concludes the case where $c=1$.

The general case proceeds by induction on $c$, replacing $R$ with its homogeneous localization at a prime containing $\mathfrak{p}$ and having dimension $\operatorname{dim}(R / \mathfrak{p})-1$.

Recall that the Bass number of a module $M$ at the prime $\mathfrak{p}$ in cohomological degree $i$ is the number of indecomposable summands isomorphic to a shift of $\underline{E}(R / \mathfrak{p})$ appearing at the $i^{\text {th }}$ stage in any minimal injective resolution of $M$. These numbers are always finite if $M$ is finitely generated, but may in general be infinite.

Corollary 3.6. Suppose $R$ has a unique maximal homogeneous ideal $\mathfrak{m}$. Let $M \in \mathcal{M}$ be a finitely generated $R$-module, and $n$ be a positive integer. Then there exists $\alpha \in Q^{\mathrm{gp}}$ such that for all $\beta \in \alpha+Q$,

1. $\check{C}(M(-\beta))=M(-\beta)$, and

2. $R^{j} \check{C}(M(-\beta))=0$ if $1 \leq j<n$.

Proof. Since $M$ is finitely generated, the Bass numbers of $M$ at $\mathfrak{m}$ are finite. Thus $\Gamma_{\mathfrak{m}} J^{i}$ is a finite direct sum of indecomposables for each $i \leq n+\operatorname{dim}(R)$. These can be moved to have nonzero $Q$-graded part by some shift $(-\alpha)$. Lemma 3.2 and Proposition 3.5 together then imply that $\check{C}$ fixes $J^{*}(-\beta)$ in cohomological degree $n$ and less for all $\beta \in \alpha+Q$.

Remark 3.7. If $R$ is a ring with only finitely many homogeneous primes (e.g., a semigroup ring), then the conclusion of Corollary 3.6 holds for any $M$ with finite Bass numbers, as then $M$ has an injective resolution with finitely many summands in each cohomological degree.

Example 3.8. Let $Q \subset \mathbb{Z}^{d}$ be a finitely generated semigroup, and let $R=$ $k[Q]$, graded by $Q$. Ishida [Ish88] constructed a dualizing complex for $R$, in which each indecomposable injective appears without shift. When $R$ is Cohen-Macaulay, this is an injective resolution of the canonical module $\omega_{R}$ that is fixed by the Cech hull. Hence Corollary 3.6 holds for $\omega_{R}$ with $\alpha=0$. It follows that for $q>0$ we have

$$
F_{2}^{p, q}\left(\omega_{R}\right)=H_{I}^{p}\left(R^{q} \check{C} \omega_{R}\right)=0,
$$

so $F\left(\omega_{R}\right)$ converges to $H_{I}^{p+q}\left(\omega_{R}\right)$. Thus $E\left(\omega_{R}\right)$ likewise converges to a filtration of $H_{I}^{p+q}\left(\omega_{R}\right)$. We will use this fact in the next section (Proposition 4.9) to compute $H_{I}^{p+q}\left(\omega_{R}\right)$ for $I$ prime.

Example 3.9. The phenomenon predicted by Corollary 3.6 is clearly illustrated in Example 2.4: As $u$ and $v$ increase, the derived functors of $\check{C}(k(-u,-v))$ in positive cohomological degrees $<u-1$ vanish. 
Theorem 3.10. Suppose $R$ has a unique homogeneous maximal ideal. Let $M$ be a finitely generated $R$-module, and $n$ be a positive integer. Then there exists $\alpha \in Q^{\mathrm{gp}}$ such that for all $\beta \in \alpha+Q$, the spectral sequence

$$
E_{2}^{p, q}(M(-\beta))=R^{p} \check{C} H_{I}^{q}(M(-\beta)) \Rightarrow H_{I}^{p+q}(M)(-\beta)
$$

converges to a local cohomology module for $p+q<n$.

Proof. Choose $\alpha$ as in Corollary 3.6. Then for all $\beta \in \alpha+Q$,

$$
F_{2}^{p, q}(M(-\beta))=H_{I}^{p}\left(R^{q} \check{C} M(-\beta)\right)=\left\{\begin{array}{ll}
0 & \text { if } q>0 \\
H_{I}^{p}(M)(-\beta) & \text { if } q=0
\end{array} .\right.
$$

Hence if $p+q<n, R^{p+q}\left(\Gamma_{I} \check{C}\right)(M(-\beta))=H_{I}^{p+q}(M(-\beta))$. Since $E(M(-\beta))$ converges to the former by Proposition 3.3, the result follows.

Injective resolutions are rarely finite, so no matter which $\alpha$ is chosen in Theorem 3.10, $F(M)$ really can converge to something other than $H_{I}^{p+q}(M)$ in large cohomological degrees. For example, if we take $Q, R$, and $k(-u,-v)$ as in Example 2.4, then $H_{I}^{i}(k(-u,-v))$ vanishes for $i \geq 1$ and any $I$. On the other hand, we have $F_{2}^{p, q}(k(-u,-v))=0$ for $p \geq 1$ and $F_{2}^{0, q}$ nonzero for $q \in\{u-1, u\}$, so the nonvanishing derived functors of $\check{C}$ cause $F(k(-u,-v))$ to fail to converge to local cohomology in these degrees.

However, since $H_{I}^{p+q}(M)$ vanishes in sufficiently high cohomological degrees, choosing $n$ large in the theorem does show how the collection of $Q$ graded parts $H_{I}^{j}(M)(-\beta)_{Q}$ for all $j$ determine the entire local cohomology modules. As we shall see in Section 4 , the $Q$-graded portion of a local cohomology module is often much easier to understand than the local cohomology module itself.

\section{Semigroup rings.}

One of the ways of understanding local cohomology $H_{I}^{*}(-)$ in terms of finitely generated modules is by taking limits (over $m$ ) of modules $\operatorname{Ext}_{R}\left(R / I^{m},-\right)$. Unfortunately, these limits are frequently quite badly behaved (see[EMS00], for example). Here, we bypass them entirely, in the case where $R=k[Q]$ is an affine semigroup algebra over a field $k$, by constructing the graded pieces of $H_{I}^{\cdot}(M)$ in terms of the derived functors $\underline{\operatorname{Ext}}_{R}\left(R / I^{m}, M\right)$ of $\underline{\operatorname{Hom}}_{R}\left(R / I^{m}, M\right)$ for a single fixed $m$, using Theorem 3.10. In order for this to work, we need to know what the $Q$-graded part of local cohomology looks like.

In Sections $4-7$, we set $R=k[Q]$, an affine semigroup algebra graded by $Q \subseteq \mathbb{Z}^{d}$, which is not assumed normal. Such a ring satisfies the hypotheses of Corollary 3.6, so that all of the machinery of the previous sections applies. For $k[Q]$ we also have a simpler expression for the Čech hull. In what follows, $Q$ is viewed as contained in $k[Q]$ via $a \mapsto \mathbf{x}^{a}$. 
Proposition 4.1. When $R=k[Q]$ and $M \in \mathcal{M}$, we have

$$
(\check{C} M)_{\beta} \cong \operatorname{Hom}_{R}\left(R_{Q+\beta}, M\right) \text {. }
$$

If $a \in Q$, we have a commutative diagram:

$$
\begin{array}{ccc}
(\check{C} M)_{\beta} & \stackrel{\cdot \mathbf{x}^{a}}{\longrightarrow} & (\check{C} M)_{\beta+a} \\
\downarrow & \downarrow & \downarrow \\
\operatorname{Hom}_{R}\left(R_{Q+\beta}, M\right) & \longrightarrow \operatorname{Hom}_{R}\left(R_{Q+a+\beta}, M\right),
\end{array}
$$

where the vertical arrows are isomorphisms and the bottom arrow is induced by the inclusion of $R_{Q+a+\beta}$ in $R_{Q+\beta}$.

Proof. By definition, $(\check{C} M)_{\beta}=\operatorname{Hom}_{R}\left(R_{Q-\beta}(-\beta), M\right)$. Multiplication by $\mathbf{x}^{\beta}$ induces an injection $R_{Q-\beta}(-\beta) \rightarrow R_{Q+\beta}$; this is an isomorphism since both of these modules are supported in the same degrees.

Moreover, one has the following commutative diagram:

$$
\begin{array}{ccc}
R_{Q-\beta-a}(-\beta-a) & \stackrel{\mathbf{x}^{a}}{\longrightarrow} & R_{Q-\beta}(-\beta) \\
\downarrow & & \downarrow \\
R_{Q+\beta+a} & \longrightarrow & R_{Q+\beta}
\end{array}
$$

from which the rest of the proposition follows immediately.

Lemma 4.2. Let $J=\underline{E}(R / \mathfrak{p})(-\alpha)$ be an indecomposable injective, and $I$ an ideal of $R$. There exists $n \in \mathbb{N}$ such that for all $m>n,\left(\Gamma_{I} J\right)_{Q}=$ $\underline{\operatorname{Hom}}_{R}\left(R / I^{m}, J\right)_{Q}$.

Proof. Suppose $I$ is not contained in $\mathfrak{p}$. Then some element of $I$ acts as a unit on $R / \mathfrak{p}$, so $\Gamma_{I} J=\underline{\operatorname{Hom}}_{R}\left(R / I^{m}, J\right)=0$ and the result is trivial. Thus it suffices to show this result for $I$ contained in $\mathfrak{p}$. In this case $\Gamma_{I} J=J$, so it suffices to show that $\underline{\operatorname{Hom}}_{R}\left(R / I^{m}, J\right)_{Q}=J_{Q}$; i.e., that every element of $J_{Q}$ is killed by $I^{m}$.

Let $\tau$ be a linear functional that takes nonnegative values on $Q$, such that if $b \in Q$, then $\tau(b)>0 \Leftrightarrow b \in \mathfrak{p}$. Then $\underline{E}(R / \mathfrak{p})$ is supported in those degrees $\beta$ such that $\tau(\beta) \leq 0$. Thus $J$ is supported in those degrees $\beta$ such that $\tau(\beta) \leq \tau(\alpha)=: n$.

Suppose $m>n$. Let $y \in J_{Q}$ and $x \in I^{m}$ be nonzero homogeneous elements of degrees $b$ and $c$, respectively. Then $\tau(b) \geq 0$ because $b \in Q$ and $\tau(c) \geq m$ because $x \in I^{m} \subseteq \mathfrak{p}^{m}$. Thus $x y$ lies in degree $b+c$ and $\tau(b+c)>n$, so $x y=0$, as required.

Now we can apply the Lemma to describe $Q$-graded parts of local cohomology.

Proposition 4.3. Let $M \in \mathcal{M}$ be finitely generated, and I be a graded ideal of $R$. Fix a nonnegative integer $i$. Then there exists $m_{0} \in \mathbb{N}$ such that for any $m \geq m_{0}$,

$$
H_{I}^{i}(M)_{Q} \cong \underline{\operatorname{Ext}}_{R}^{i}\left(R / I^{m}, M\right)_{Q}
$$


Proof. Let $J^{\cdot}$ be an injective resolution for $M$, and choose $m_{0}$ sufficiently large that $\left(\Gamma_{I} N\right)_{Q}=\underline{\operatorname{Hom}}_{R}\left(R / I^{m}, N\right)_{Q}$ agree for every $m \geq m_{0}$ and every indecomposable injective summand $N$ appearing in cohomological degree $i$ or lower in $J^{*}$. Then the first $i$ right derived functors of $\left(\Gamma_{I}-\right)_{Q}$ and $\underline{\operatorname{Hom}}_{R}\left(R / I^{m},-\right)_{Q}$ agree on $M$; since $(-)_{Q}$ is exact this means $H_{I}^{i}(M)_{Q} \cong$ $\underline{\operatorname{Ext}}_{R}^{i}\left(R / I^{m},-\right)_{Q}$.

Example 4.4. If $Q$ is saturated and $M=\omega_{R}$ then the power of $I$ in Proposition 4.3 can be set equal to 1 ; i.e.,

$$
\operatorname{Ext}_{R}^{p}\left(R / I, \omega_{R}\right)_{Q} \cong H_{I}^{p}\left(\omega_{R}\right)_{Q}
$$

since the indecomposable summands of the injective resolution of $\omega_{R}$ are unshifted $\underline{E}(R / \mathfrak{p})$ 's.

Corollary 4.5. If $M \in \mathcal{M}$ is finitely generated, $H_{I}^{i}(M)_{Q}$ is finitely generated.

Corollary 4.6. Let $M \in \mathcal{M}$ be finitely generated. Then $H_{I}^{i}(M)$ has a finitely generated essential submodule if and only if for some $\beta \in Q^{\mathrm{gp}}$ the natural map $H_{I}^{i}(M)(-\beta) \rightarrow \check{C}\left(H_{I}^{i}(M)(-\beta)\right)$ is an injection.

Proof. Suppose $H_{I}^{i}(M)$ has a finitely generated essential submodule $N$. Then we can shift $N$ so that all of its generators have degrees in $Q$; i.e., there exists $\beta$ such that $N(-\beta) \subset\left(H_{I}^{i}(M)(-\beta)\right)_{Q}$. Since the map $H_{I}^{i}(M)(-\beta) \rightarrow$ $\check{C} H_{I}^{i}(M)(-\beta)$ is injective on its $Q$-graded part, it is injective on $N(-\beta)$; since $N(-\beta)$ is essential the map is injective everywhere.

Conversely, $H_{I}^{i}(M)(-\beta)_{Q}$ is an essential submodule of $\check{C}\left(H_{I}^{i}(M)(-\beta)\right)$ and hence of $H_{I}^{i}(M)(-\beta)$. By Corollary 4.5 it is finitely generated.

The upshot of the above is that since the spectral sequence $E(M)$ of Proposition 3.3 depends only on the $Q$-graded parts of the local cohomology modules whic appear in it, we can just replace these local cohomology modules with the corresponding Ext-modules.

Theorem 4.7. Let $M$ be a finitely generated module over $R=k[Q]$, and $n$ be a positive integer. Then there exists $\alpha \in Q^{\mathrm{gp}}$ such that for all $\beta \in \alpha+Q$, there exists $m \in \mathbb{Z}$ making the spectral sequence

$$
E_{2}^{p, q}(M(-\beta))=R^{p} \check{C} \underline{\operatorname{Ext}}_{R}^{q}\left(R / I^{m}, M(-\beta)\right) \Rightarrow H_{I}^{p+q}(M)(-\beta)
$$

from Proposition 3.3 converge to a local cohomology module for $p+q<n$. Taking degree $\gamma$ parts for any $\gamma \in Q^{\mathrm{gp}}$ yields a spectral sequence of iterated Ext modules,

$$
E_{2}^{p, q}(M(-\beta))_{\gamma}=\operatorname{Ext}_{R}^{p}\left(R_{Q+\gamma}(\beta), \underline{\operatorname{Ext}}_{R}^{q}\left(R / I^{m}, M\right)\right) \Rightarrow H_{I}^{p+q}(M)_{\gamma-\beta} .
$$

Proof. This is immediate from Theorem 3.10 and Proposition 4.3. 
Example 4.8. Returning to the setting of Example 3.4, we find using this theorem that if $M$ is a $\mathbb{Z}^{d}$-graded module over a polynomial ring in $d$ variables and $I$ is a monomial ideal, then there exist $m \in \mathbb{Z}$ and $\beta \in \mathbb{Z}^{d}$ such that $H_{I}^{i}(M)=\check{C} \underline{\operatorname{Ext}}_{R}^{i}\left(R / I^{m}, M(-\beta)\right)$. This generalizes a result proved independently by Mustaţă [Mus00] and Terai [Ter99].

For saturated $Q$, Theorem 4.7 takes an especially nice form for canonical modules. Recall that a face of $Q$ is the set of degrees of elements outside a prime ideal of $R$.

Proposition 4.9. Suppose $R$ is normal and of dimension $d$. Let $\mathfrak{p}$ be a prime of $R$, corresponding to an $n$-dimensional face of $Q$. Then $H_{\mathfrak{p}}^{d-i}\left(\omega_{R}\right) \cong$ $R^{n-i} \check{C}\left(\omega_{R / \mathfrak{p}}\right)$.

Proof. $R^{q} \check{C} \underline{\operatorname{Ext}}_{R}^{p}\left(R / \mathfrak{p}, \omega_{R}\right) \Rightarrow H_{\mathfrak{p}}^{p+q}\left(\omega_{R}\right)$ by Example 4.4 and Theorem 4.7. Since $R / \mathfrak{p}$ is a dimension $n$ Cohen-Macaulay quotient of the Cohen-Macaulay ring $R$ of dimension $d$, the module $\operatorname{Ext}_{R}^{p}\left(R / \mathfrak{p}, \omega_{R}\right)$ is nonzero only when $p=d-n$, in which case it is $\omega_{R / \mathfrak{p}}$. Thus the spectral sequence degenerates, and $R^{q} \check{C}\left(\omega_{R / \mathfrak{p}}\right) \cong H_{\mathfrak{p}}^{q+d-n}\left(\omega_{R}\right)$.

Example 4.10. Let $Q$ be the semigroup on four generators $\{x, y, u, v\}$ and one relation $x+u=y+v$, and $R=k[Q]$. In [Har70], Hartshorne shows that for the ideal $I=\left(\mathbf{x}^{u}, \mathbf{x}^{v}\right)$, the local cohomology module $H_{I}^{1}\left(\omega_{R}\right)$ has a finitely generated essential submodule while $H_{I}^{2}\left(\omega_{R}\right)$ has an infinite dimensional socle, supported in degrees $n(x-v)$ for $n>0$. This is consistent with Proposition 4.9, which says that infinite-dimensional socles must arise from a nonvanishing higher derived functor of the Čech hull. See Section 7 for a combinatorial explanation of why this bad behavior occurs, in the context of its generalization to arbitrary affine semigroup rings.

\section{Finiteness for simplicial semigroups.}

Letting $Q \subseteq \mathbb{Z}^{d}$ be affine as in the previous section, the above machinery allows us to make strong statements about local cohomology over $R=k[Q]$. Indeed, the fact that local cohomology modules "come from" the derived functors of the Čech hull forces certain structure on them. This structure is codified in the notion of a straight module, a common generalization of notions due to Miller [Mil00] (who defined a-determined modules over a polynomial ring for $\mathbf{a} \in \mathbb{N}^{n}$ ) and Yanagawa [Yan01, Yan00] (who defined straightness for a restrictive class of modules over semigroup rings). One obtains from Theorem 4.7 that local cohomology modules over semigroup rings are straight when shifted appropriately. Over a simplicial (and not necessarily normal) semigroup ring this forces them to have finite Bass numbers (Theorem 5.8). The key to all this is the following definition: 
Definition 5.1. Let $Q \subseteq \mathbb{Z}^{d}$ be affine and $R=k[Q]$. The category $\mathcal{S}$ of straight $R$-modules is the smallest subcategory of the $Q^{\mathrm{gp}}$-graded modules $\mathcal{M}$ such that (1) all indecomposable injectives $J$ satisfying $J_{Q} \neq 0$ are in $\mathcal{S}$; (2) finite direct sums of modules in $\mathcal{S}$ are in $\mathcal{S}$; and (3) if $\phi$ is a homomorphism of straight modules, then $\operatorname{ker}(\phi)$ and $\operatorname{coker}(\phi)$ are straight.

Proposition 5.2. Let $M \in \mathcal{M}$ be finitely generated. Then $R^{i} \check{C} M$ is straight.

Proof. By definition, a finite direct sum of straight modules is straight. Thus if $C^{\bullet}$ is a complex of finite direct sums of indecomposable injectives, and each indecomposable injective in $C^{\bullet}$ has nontrivial $Q$-graded part, then $C^{\cdot}$ is a complex of straight modules, and the cohomology of $C^{\bullet}$ is straight. In particular, if $C^{\cdot}=\check{C} J^{\cdot}$ is the Čech hull of an injective resolution $J^{\bullet}$ of $M$, then $\check{C} J^{\bullet}$ is a complex of straight modules by Lemma 3.2. Thus its cohomology is straight, as required.

Corollary 5.3. Let $M \in \mathcal{M}$ be finitely generated. Then there exists an element $a \in Q$ such that $H_{I}^{i}(M)(-a)$ is straight.

Proof. By Theorem 4.7 and Proposition 5.2, we have $a \in Q$ such that $H_{I}^{i}(M)(-a)$ is the limit of a spectral sequence of straight modules. This spectral sequence yields a finite filtration of $H_{I}^{i}(M)(-a)$ whose associated graded modules are therefore straight.

Straight modules have a number of useful properties. In particular, the fact that they can be "built out of" indecomposable injectives with nontrivial $Q$-graded part by taking kernels, cokernels, and finite direct sums forces many of their graded pieces to be isomorphic to each other.

Proposition 5.4. Let $M$ be a straight module over $R=k[Q]$. Then:

1. $M_{Q}$ is finitely generated.

2. $M(-a)$ is straight for all $a \in Q$.

3. Multiplication by $\mathbf{x}^{a}$ is an isomorphism $M_{\beta} \rightarrow M_{a+\beta}$ whenever $\beta \in$ $Q^{\mathrm{gp}}$ and $a \in Q$ satisfy $(\beta+Q) \cap Q=(a+\beta+Q) \cap Q$.

Proof. If the above three properties hold for $M$ and $N$, then they also hold for $M \oplus N$, as well as $\operatorname{ker}(\phi)$ and $\operatorname{coker}(\phi)$ for any $\phi: M \rightarrow N$. Thus it suffices to check that if $J$ is an indecomposable injective, and $J_{Q}$ is nonzero, then $J$ has the above properties. $J_{Q}$ is clearly finitely generated, and if $J_{Q}$ is nonzero, so is $J(-a)_{Q}$, so the first two properties are clear.

For the third property, note that $\check{C} J=J$ by Lemma 3.2 . Thus in particular,

$$
\begin{aligned}
J_{\beta} & =(\check{C} J)_{\beta}=\operatorname{Hom}_{R}\left(R_{Q+\beta}, J\right) \\
\text { and } J_{a+\beta} & =(\check{C} J)_{a+\beta}=\operatorname{Hom}_{R}\left(R_{Q+a+\beta}, J\right) .
\end{aligned}
$$


The hypothesis in part 3 says that $R_{Q+\beta}=R_{Q+a+\beta}$, whence multiplication by $\mathbf{x}^{a}$ is an isomorphism $J_{\beta} \rightarrow J_{a+\beta}$, as required.

The third property of Proposition 5.4 motivates the following definition: Definition 5.5. An essential point for $Q$ is an element $\varepsilon \in Q^{\text {gp }}$ such that if $a \in Q$ and $(\varepsilon+Q) \cap Q=(a+\varepsilon+Q) \cap Q$, then $a$ is a unit. The essential set $\mathcal{E}$ is the $Q$-set generated by the essential points; i.e., $\mathcal{E}$ is the union $\bigcup(Q+\varepsilon)$ over essential points $\varepsilon$.

Now we derive the main theorem of this section, Theorem 5.8, from combinatorial properties of the essential set, which we develop in detail in Section 6. The key results from that section which we use below are Lemma 6.6, which is an existence result for essential points, and Proposition 6.13, which allows us to control the size of the essential set when $Q$ has simplicial saturation.

Proposition 5.6. If $M$ is straight, then $M_{\mathcal{E}}$ is an essential submodule of $M$.

Proof. Suppose $0 \neq x \in M_{\alpha}$, and choose an essential point $\varepsilon$ with $\varepsilon-\alpha \in Q$ and $(\varepsilon+Q) \cap Q=(\alpha+Q) \cap Q$, using Lemma 6.6. Proposition 5.4 shows that multiplication by $\mathbf{x}^{\varepsilon-\alpha}$ is an isomorphism $M_{\alpha} \rightarrow M_{\varepsilon}$. Thus any submodule of $M$ containing $x$ contains $\mathbf{x}^{\varepsilon-\alpha} x \in M_{\mathcal{E}}$.

Observe that the results in this section so far have used no extra hypotheses on the affine semigroup $Q$. Since our goal involves simplicial semigroups, this will change starting now.

Proposition 5.7. Let $Q$ be an affine semigroup whose saturation is simplicial modulo units, and $M$ a straight module over $k[Q]$. Then the Bass numbers of $M$ are finite.

Proof. Taking $a$ as in Proposition 6.13, we find that $M_{\mathcal{E}} \subset M_{Q-a}$, so $M_{\mathcal{E}}(-a) \subset(M(-a))_{Q}$. Since $M(-a)$ is straight, $M(-a)_{Q}$ is finitely generated. Thus $M_{\mathcal{E}}$ is finitely generated, so $M$ has a finitely generated essential submodule by Proposition 5.6. In particular, its Bass numbers in cohomological degree zero are finite. Moreover, if $J^{\bullet}$ is a minimal injective resolution of $M$, then $J^{0}(-a)$ is straight because it has a $Q$-graded essential submodule $M(-a)_{Q}$. Therefore $\operatorname{coker}\left(M(-a) \rightarrow J^{0}(-a)\right)$ is straight, whence the result follows by induction on the cohomological degree.

The Bass numbers of such an $M$ at ungraded primes are also finite, by results of [GW78].

Theorem 5.8. Let $Q$ be an affine semigroup whose saturation is simplicial modulo units. If $M$ is a finitely generated $Q^{\mathrm{gp}}$-graded $k[Q]$-module, then the Bass numbers of $H_{I}^{i}(M)$ are finite, for any $Q$-graded ideal $I$. 
Proof. This is immediate from Proposition 5.7 and Corollary 5.3.

Corollary 5.9. Suppose $Q$ is affine, with $Q^{\text {sat }}$ simplicial modulo units, and let $M \in \mathcal{M}$ be finitely generated over $R=k[Q]$. Then there exist $\beta \in Q^{\mathrm{gp}}$ and $n \in \mathbb{N}$ such that

$$
H_{I}^{i}(M)(-\beta) \cong \check{C} \underline{\operatorname{Ext}}_{R}^{i}\left(R / I^{n}, M(-\beta)\right) .
$$

Proof. Since the Bass numbers of $H_{I}^{i}(M)$ are finite, by Remark 3.7 there exists $\beta$ such that $H_{I}^{i}(M)(-\beta)$ is fixed by the Cech hull. Then $H_{I}^{i}(M)(-\beta)$ is the Cech hull of its $Q$-graded part and the result follows from Proposition 4.3.

\section{The essential set.}

The essential set, introduced in Definition 5.5, fleshes out in some detail the combinatorics hidden in an affine semigroup. Since we believe this combinatorics is of independent interest, we determine in Theorem 6.2, Proposition 6.4, and the comments in between, the structure of the essential set in the saturated case, along with its relation to Hilbert bases, monomial modules, irrelevant ideals of toric varieties, and Alexander duality. The rest of the section we devote to providing the necessary relations between essential sets of unsaturated semigroups and those of their saturations, including the results already applied in Section 5 .

We need a bit of notation. Associated to an affine semigroup $Q$ are its facets $F_{1}, \ldots, F_{r}$; these are the degrees of homogeneous elements outside of the $r$ codimension-one $Q$-graded primes of $k[Q]$. There are unique primitive integer-valued linear functionals $\left\{\tau_{1}, \ldots, \tau_{r}\right\}$ on $Q^{\mathrm{gp}}$, nonnegative on $Q$, such that $F_{i}=\left\{b \in Q \mid \tau_{i}(b)=0\right\}$. Given $\alpha \in Q^{\text {gp }}$, define $\tau(\alpha) \in \mathbb{Z}^{r}$ to be the vector $\left(\tau_{1}(\alpha), \ldots, \tau_{r}(\alpha)\right)$, and let $\tau(\alpha)^{+}$be the vector obtained from $\tau(\alpha)$ by replacing its negative entries with zeros.

Lemma 6.1. Suppose $Q$ is saturated. Then $(\alpha+Q) \cap Q=(\beta+Q) \cap Q$ if and only if $\tau(\alpha)^{+}=\tau(\beta)^{+}$. In particular, $\varepsilon$ is an essential point if and only if $\tau(\varepsilon)^{+} \neq \tau(a+\varepsilon)^{+}$for all nonunits a in some generating set for $Q$.

Proof. Since $Q$ is saturated, $(\alpha+Q) \cap Q$ is the set of lattice points $\gamma \in Q^{\text {gp }}$ inside the polyhderon defined by $\left\{\tau_{i}(\gamma) \geq 0\right.$ and $\left.\tau_{i}(\gamma) \geq \tau_{i}(\alpha) \mid i=1, \ldots, r\right\}$. This is the polyhedron defined by the inequalities $\left\{\tau_{i}(\gamma) \geq \tau(\alpha)_{i}^{+} \mid i=\right.$ $1, \ldots, r\}$, and the first claim follows easily.

The map $\tau: Q^{\text {gp }} \rightarrow \mathbb{Z}^{r}$ takes $Q$ to the semigroup $\tau(Q)$ isomorphic to the quotient of $Q$ by its group of units. As a consequence, the definition of essential point translates to: $\varepsilon$ is an essential point if and only if $\tau(\varepsilon)^{+} \neq$ $\tau(a+\varepsilon)^{+}$for all nonunits $a \in Q$. But since $\tau_{i}$ is nonnegative on $Q$ for all $i$, the second statement follows. 
The lack of nontrivial units in $\tau(Q)$ endows it with a unique minimal set $\mathcal{H}$ of semigroup generators, called the Hilbert basis of $\tau(Q)$. Each element of $\mathcal{H}$ imposes a condition that $\alpha$ must satisfy to be essential. To express this condition, define, for $h \in \mathbb{N}^{r}$, the set $\langle h\rangle=\left\{\zeta \in \mathbb{Z}^{r} \mid \zeta_{i}>-h_{i}\right.$ for some $i$ such that $\left.h_{i}>0\right\}$. Observe that $\langle h\rangle$ is a union of half-spaces, and is defined in such a way that $\tau^{-1}(\langle\tau(a)\rangle)=\left\{\varepsilon \in Q^{\text {gp }} \mid \tau(\varepsilon)^{+} \neq \tau(\varepsilon+a)^{+}\right\}$.

Theorem 6.2. If $Q$ is saturated then the essential set $\mathcal{E}$ consists entirely of essential points. Furthermore, $\mathcal{E}=\tau^{-1}\left(\bigcap_{h \in \mathcal{H}}\langle h\rangle\right)=\bigcap_{h \in \mathcal{H}} \tau^{-1}(\langle h\rangle)$.

Proof. The second sentence follows from Lemma 6.1 and the remarks following it because $\tau^{-1}(\mathcal{H})$ generates $Q$. Since $\langle h\rangle$ is stable under the action of $Q, \tau^{-1}(\langle h\rangle)$ is $Q$-stable, too. Thus the essential points already form a $Q$-set, which therefore equals $\mathcal{E}$.

Example 6.3. We consider once again the semigroup $Q$ generated by three elements $x, y, z$ such that $x+y=2 z$. $\tau$ embeds $Q$ in $\mathbb{Z}^{2}$ by sending $x$ to $(2,0), y$ to $(0,2)$, and $z$ to $(1,1)$; the image of $Q^{\text {gp }}$ in $\mathbb{Z}^{2}$ is the sublattice of index 2 consisting of pairs $(u, v)$ such that $u$ and $v$ have the same parity.

The points $(0,2),(1,1)$ and $(2,0)$ form a Hilbert basis for $\tau(Q)$. $\langle(0,2)\rangle$ consists of those points $(u, v)$ in $\mathbb{Z}^{2}$ with $v \geq-1$; similarly $\langle(2,0)\rangle$ consists of those points with $u \geq-1$. Finally $\langle(1,1)\rangle$ consists of points with either $u$ or $v$ nonnegative. Thus the essential points are those points $\alpha \in Q^{\mathrm{gp}}$ such that $\tau(\alpha)$ has one of the following forms:

1. $(-1, v)$ for some odd positive $v$.

2. $(u,-1)$ for some odd positive $u$.

3. $(u, v)$ for $u, v$ nonnegative and with the same parity.

Figure 1 shows the essential set embedded in $\mathbb{Z}^{2}$ via $\tau$. The spots (both hollow and solid) represent elements of $Q^{\mathrm{gp}}$; solid spots are essential points. The regions in which $\alpha+Q \cap Q$ remain constant are enclosed by dotted lines. Note in particular that there is an essential point in every region, and that the essential points form a $Q$-set, as predicted by the theorem.

If we refer back to Example 2.4, we see that the socles of $R^{p} \check{C}$ computed there lie within the essential set, as Proposition 5.6 and Proposition 5.2 predict. Also note that $z+\mathcal{E} \subset Q$. One will be able to translate $\mathcal{E}$ so that it lies in $Q$ precisely when $Q$ is simplicial; this is the content of Proposition 6.13, which is the central goal of this section.

The essential set $\mathcal{E}$ is related to a number of other notions already playing roles in the study of semigroup algebras and toric varieties. For instance, the subset $\langle\mathcal{H}\rangle:=\bigcap_{h \in \mathcal{H}}\langle h\rangle$ of $\mathbb{Z}^{r}$ is a monomial module [BS98], so $\mathcal{E}$ might be called a skew monomial module inside the lattice $Q^{\mathrm{gp}}$. To get a better picture, $\langle\mathcal{H}\rangle \subset \mathbb{Z}^{r}$ is a "fuzzy neighborhood" of a certain union $\mathcal{U}$ of orthants, in the sense that there is a vector $z \in \mathbb{N}^{r}$ such that $\mathcal{U} \subseteq\langle\mathcal{H}\rangle \subseteq \mathcal{U}-z$. In fact, 


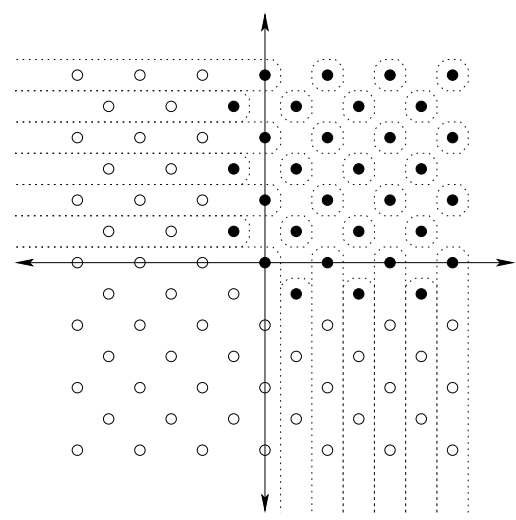

Figure 1. The essential set of the semigroup generated by $(2,0),(1,1)$, and $(0,2)$.

each set $\langle h\rangle$ contains and approximates the union $U_{h}=\bigcup_{h_{i}>0}\left\{\zeta \in \mathbb{Z}^{r} \mid \zeta_{i} \geq\right.$ $0\}$ of half-spaces, and $\mathcal{U}=\bigcap_{h \in \mathcal{H}} U_{h}$; our $z$ can be any vector with $z_{i}>h_{i}$ for all $h \in \mathcal{H}$ and all $i$.

We can get an even better handle on $\mathcal{U}$ in the case where $Q$ is the cone over $\bar{Q}$, an integral polytope in $\mathbb{Z}^{d-1} \times\{1\} \subset \mathbb{Z}^{d-1} \times \mathbb{Z}=\mathbb{Z}^{d}$. For each face $F$ of $Q$, let $\operatorname{van}(F) \subseteq\{1, \ldots, r\}$ be the indices of functionals vanishing on $F$; similarly, for $z \in \mathbb{N}^{r}$, let $\operatorname{van}(z)=\left\{i \in\{1, \ldots, r\} \mid z_{i}=0\right\}$. For instance, if $F=F_{i}$ is a facet then $\operatorname{van}\left(F_{i}\right)=\{i\}$, and $\operatorname{van}(z)=\operatorname{van}(F)$ if and only if $z_{i}=0$ and $z_{j}>0$ for all $j \neq i$. The polynomial ring $k\left[\mathbb{N}^{r}\right]$ is the Cox homogeneous coordinate ring [Cox95] of the projective toric variety $X$ whose isomorphism class and embedding in projective space are determined by $\bar{Q}$. The Cox ring comes equipped with the irrelevant ideal $B=\left\langle\mathbf{x}^{z}\right| z \in$ $\mathbb{N}^{r}$ and $\operatorname{van}(z) \subseteq \operatorname{van}(F)$ for some face $F$ of $\left.Q\right\rangle$.

Proposition 6.4. If $\check{C}$ is the Čech hull over $\mathbb{N}^{r}$, then $-\zeta \in \mathcal{U}$ if and only if $\mathbf{x}^{\zeta} \notin \check{C}(B)$. Equivalently, the $k\left[\mathbb{N}^{r}\right]$-submodule $\left\langle\mathbf{x}^{\zeta} \mid \zeta \in \mathcal{U}\right\rangle \subset k\left[\mathbb{Z}^{r}\right]$ is the shift by $(1, \ldots, 1) \in \mathbb{Z}^{r}$ of the $\check{C}$ ech hull $\check{C}\left(B^{\star}\right)$ of the ideal $B^{\star}$ Alexander dual to $B$ [ER98], [MP01, Lecture VI].

In the case where $\bar{Q}$ is a simple polytope, so the corresponding projective toric variety is simplicial, $B^{\star}$ is the Stanley-Reisner ideal for the simplicial polytope polar to $\bar{Q}$.

Proof. The equivalence of the two statements is [Mil98, Lemma 2.11]. Note that $\check{C}(B)$ is, a priori, a submodule of $k\left[\mathbb{Z}^{r}\right]$ since the latter is the injective hull of $B$ and the former is an essential extension. Now $\mathbf{x}^{\zeta} \in \check{C}(B)$ if and only if $\operatorname{van}\left(\zeta^{+}\right) \subseteq \operatorname{van}(F)$ for some face $F$ by Remark 2.3. On the other hand, $-\zeta \in U_{h}$ precisely when $\zeta_{i} \leq 0$ for some $i$ with $h_{i}>0$; that is, when $\operatorname{van}\left(\zeta^{+}\right) \nsubseteq \operatorname{van}(h)$. Therefore, $-\zeta \notin \mathcal{U}$ if and only if $\operatorname{van}\left(\zeta^{+}\right) \subseteq \operatorname{van}(h)$ for 
some $h \in \mathcal{H}$. This occurs if and only if $\operatorname{van}\left(\zeta^{+}\right) \subseteq \operatorname{van}(F)$ for some face $F$, because: $\operatorname{van}(F) \subseteq \operatorname{van}(h)$ for all $h \in F$; and each $h \in \mathcal{H}$ lies in the relative interior of some $F$, so $\operatorname{van}(h)=\operatorname{van}(F)$ for this $F$. We conclude that $\mathbf{x}^{\zeta} \in \check{C}(B)$ if and only if $-\zeta \notin \mathcal{U}$.

Example 6.5. We illustrate this for the semigroup $Q$ of Example 4.10; that is, the semigroup on four generators $\{x, y, u, v\}$ with the relation $x+u=y+$ $v$. If we order the four facets appropriately, $\tau$ embeds $Q^{\mathrm{gp}}$ in $\mathbb{Z}^{4}$ by sending $x$ to $(1,0,0,1), y$ to $(0,1,0,1), u$ to $(0,1,1,0)$ and $v$ to $(1,0,1,0)$. The image $\tau\left(Q^{\mathrm{gp}}\right)$ is the lattice consisting of points $(a, b, c, d)$ with $a+c=b+d$.

Now $\langle(1,0,0,1)\rangle$ is the set of $(a, b, c, d)$ in $\mathbb{Z}^{4}$ such that either $a$ or $d$ is nonnegative. Thus, if $\alpha \in Q^{\mathrm{gp}}$ is an essential point, with $\tau(\alpha)=(a, b, c, d)$, then either $a$ or $d$ is nonnegative. Similarly, using the other elements of the Hilbert basis, we find that:

- Either $b$ or $d$ is nonnegative.

- Either $b$ or $c$ is nonnegative.

- Either $a$ or $c$ is nonnegative.

Note that in this example, the irrelevant ideal $B$ of Proposition 6.4 is generated by the elements $\mathbf{x}^{(1,0,0,1)}, \mathbf{x}^{(0,1,0,1)}, \mathbf{x}^{(0,1,1,0)}$, and $\mathbf{x}^{(1,0,1,0)}$ in the polynomial ring $k\left[\mathbb{N}^{4}\right]$. The Čech hull of this ideal is thus supported precisely on those $(a, b, c, d) \in \mathbb{Z}^{4}$ such that at least one of the pairs $\{a, d\},\{b, d\}$, $\{b, c\},\{a, c\}$ consists of strictly positive integers. Therefore, an element of $\mathbb{Z}^{4}$ fails to be in this support if and only if its negative satisfies the above four conditions. To summarize, those elements $\alpha$ of $Q^{\mathrm{gp}}$ such that $-\tau(\alpha)$ is not in the support of $\check{C} B$ are essential points, as Proposition 6.4 predicts.

The conditions on $\tau(\alpha)$ given above, together with the fact that $(a, b, c, d)$ $=\tau(\alpha)$ (and therefore $a+b=c+d$ ), imply that $\alpha$ is an essential point if (and only if) at most one of $\{a, b, c, d\}$ is negative. Note that no finite shift will take all of the essential points inside of $Q$, since one has essential points $\alpha$ whose negative coordinate is $-n$ for any natural number $n$. In particular, the degrees of the socle elements of $H_{(x, u)}^{2}\left(\omega_{R}\right)$ produced in Example 4.10 are a set of essential points whose negative coordinates are unbounded below.

More generally, the fact that we cannot shift the essential set into $Q$ means that we cannot rule out the possibility of local cohomology having infinite Bass numbers. In fact, we will construct a local cohomology module with infinite Bass numbers whenever the essential set cannot be shifted into $Q$ (Corollary 7.5).

During our proof of Theorem 5.8, we needed certain results about the structure of $\mathcal{E}$. In order to obtain them in the generality we used in Section 5 , we no longer assume that $Q$ is saturated. We begin with a lemma used in the proof of Proposition 5.6. 
Lemma 6.6. Given $\alpha \in Q^{\mathrm{gp}}$, there exists some essential point $\varepsilon$ with $(\varepsilon+$ $Q) \cap Q=(\alpha+Q) \cap Q$.

Proof. Any element $\beta \in Q^{\text {gp }}$ satisfying $(\beta+Q) \cap Q=(\alpha+Q) \cap Q$ must also satisfy $\tau(\beta) \preceq \tau(\gamma)$ for all $\gamma \in(\alpha+Q) \cap Q$, where $\preceq$ is the partial order by componentwise comparison. The set of possibilities for $\tau(\beta) \in \mathbb{Z}^{r}$ satisfying this condition is bounded above, and thus has a maximal element $\tau(\varepsilon)$. Moreover, $\tau(\beta)=\tau(\varepsilon)$ for some $\beta \in Q^{\text {gp }}$ if and only if $\beta-\varepsilon$ is a unit of $Q$. This proves that $\varepsilon$ is an essential point.

The major combinatorial result used in the previous section is the fact that if $Q^{\text {sat }}$ is (modulo its units) simplicial, then $\mathcal{E}$ can be shifted inside of $Q$. Therefore, we want an analog to Lemma 6.1 which holds even for semigroups which are not saturated. The key tool relating the combinatorics of a semigroup to the combinatorics of its normalization is provided by the next lemma. Recall that a face of $Q$ is the set of degrees of elements outside a prime ideal of $k[Q]$.

Lemma 6.7. Let $F$ be a face of $Q$. There exists $a_{F} \in F$ such that $a_{F}+Q^{F} \subset$ $Q$, where $Q^{F}:=\left(Q+F^{\mathrm{gp}}\right) \cap Q^{\text {sat }}$ is the partial saturation of $Q$ at $F$.

Proof. Let $R^{\prime}=k\left[Q^{F}\right]$ and $\widetilde{R}=k\left[Q^{\text {sat }}\right]$. Then, letting $\mathfrak{p} \subset R=k[Q]$ be the prime ideal such that $R / \mathfrak{p}=k[F]$, the $R$-algebra $R^{\prime}$ is the intersection $R_{(\mathfrak{p})} \cap \widetilde{R}$ of the homogeneous localization at $\mathfrak{p}$ with the normalization. The Lemma calls for a homogeneous element outside of $\mathfrak{p}$ to be in the conductor ideal

$$
\operatorname{ann}_{R}\left(R^{\prime} / R\right)=\left\{x \in R \mid x R^{\prime} \subset R\right\} .
$$

Such an element exists precisely when $\operatorname{ann}_{R}\left(R^{\prime} / R\right)_{(\mathfrak{p})}=R$; i.e., when the localizations $R_{(\mathfrak{p})}$ and $R^{\prime} \otimes_{R} R_{(\mathfrak{p})}$ are equal. But

$$
\begin{aligned}
R^{\prime} \otimes_{R} R_{(\mathfrak{p})} & =R_{(\mathfrak{p})} \cap\left(\widetilde{R} \otimes_{R} R_{(\mathfrak{p})}\right) \\
& =R_{(\mathfrak{p})} \cap \widetilde{R}_{(\mathfrak{p} \widetilde{R})} \\
& =R_{(\mathfrak{p})}
\end{aligned}
$$

because $R_{(\mathfrak{p})} \subseteq \widetilde{R}_{(\mathfrak{p} \widetilde{R})}$.

Remark 6.8. When $F=Q$, then $Q^{F}=Q^{\text {sat }}$, and this is the well-known fact that every semigroup $Q$ contains an element $a$ with $a+Q^{\text {sat }} \subset Q$.

With Lemma 6.7 in hand, we can now find a sufficient condition under which $(\beta+Q) \cap Q=(a+\beta+Q) \cap Q$. Let $\operatorname{van}_{\tau}(F) \subseteq\left\{\tau_{1}, \ldots, \tau_{r}\right\}$ be the subset consisting of functionals vanishing on the face $F$ of $Q$.

Lemma 6.9. Let $a \in F$, and suppose we have $\beta \in Q^{\text {gp }}$ such that $\tau_{i}(a+$ $\left.a_{F}+\beta\right) \leq 0$ for all $\tau_{i} \notin \operatorname{van}_{\tau}(F)$. Then $(\beta+Q) \cap Q=(a+\beta+Q) \cap Q$. 
Proof. Noting that $\tau_{j}(a)=\tau_{j}\left(a_{F}\right)=0$ for $\tau_{j} \in \operatorname{van}_{\tau}(F)$, the hypothesis on $a+a_{F}+\beta$ implies that the intersections with $Q$ are contained in $\left(a+a_{F}+\right.$ $\left.\beta+Q^{\text {sat }}\right)$. Therefore, it is enough to show that

$$
(\beta+Q) \cap\left(a+a_{F}+\beta+Q^{\text {sat }}\right)=(a+\beta+Q) \cap\left(a+a_{F}+\beta+Q^{\text {sat }}\right) .
$$

This follows by adding $\beta$ or $a+\beta$ to both sides of the equality in Lemma 6.10, below, and setting respectively $b=a+a_{F}$ or $b=a_{F}$.

Lemma 6.10. If $b \in F$ and $b+Q^{F} \subseteq Q$, then $Q \cap\left(b+Q^{\text {sat }}\right)=b+Q^{F}$.

Proof. We show $Q \cap\left(b+Q^{\text {sat }}\right)=\left(b+Q^{F}\right) \cap\left(b+Q^{\text {sat }}\right)$, which obviously equals $b+Q^{F}$. Now $Q \cap\left(b+Q^{\text {sat }}\right) \supseteq\left(b+Q^{F}\right) \cap\left(b+Q^{\text {sat }}\right)$, because $Q$ contains $b+Q^{F}$; and $Q \cap\left(b+Q^{\text {sat }}\right) \subseteq\left(b+Q^{F}\right) \cap\left(b+Q^{\text {sat }}\right)$, because $a-b \in Q^{F}$ when $a \in Q \cap\left(b+Q^{\text {sat }}\right)$, by definition of $Q^{F}$.

We are now in a position to state and prove the unsaturated analog of Lemma 6.1.

Proposition 6.11. Choose $a_{Q}$ so that $\tau_{i}\left(a_{Q}\right) \geq \tau_{i}\left(a_{F}\right)$ for all $i$ and $F$. Suppose $a \in Q, \beta \in Q^{\mathrm{gp}}$, and $\tau\left(a_{Q}+\beta\right)^{+}=\tau\left(a+a_{Q}+\beta\right)^{+}$. Then $(\beta+$ $Q) \cap Q=(a+\beta+Q) \cap Q$.

Proof. Let $F$ be the smallest face of $Q$ containing $a$. Then for all $\tau_{i}$ not vanishing on $F$, we have $\tau_{i}(a)>0$, so $\tau_{i}\left(a+a_{Q}+\beta\right) \leq 0$ (as otherwise the $i^{\text {th }}$ coordinates of $\tau\left(a_{Q}+\beta\right)^{+}$and $\tau\left(a+a_{Q}+\beta\right)^{+}$are unequal). Thus $\tau_{i}(a+\beta) \leq \tau_{i}\left(-a_{Q}\right) \leq-\tau_{i}\left(a_{F}\right)$, and by Lemma 6.9 we have $(\beta+Q) \cap Q=$ $(a+\beta+Q) \cap Q$, as required.

The approximation to Theorem 6.2 in the unsaturated case is as follows:

Corollary 6.12. Let $\mathcal{H}$ be the Hilbert basis for $\tau(Q)$, and $a_{Q}$ be as in Proposition 6.11. Then $\mathcal{E}+a_{Q} \subseteq \bigcap_{h \in \mathcal{H}} \tau^{-1}(\langle h\rangle)$.

Proof. Pick, for each $h \in \mathcal{H}$, an element $q_{h} \in Q$ with $\tau\left(q_{h}\right)=h$. Suppose $\varepsilon$ is an essential point. Setting $\beta=\varepsilon$ and $a=q_{h}$ in Proposition 6.11, we have $\tau\left(\varepsilon+a_{Q}\right)^{+} \neq \tau\left(\varepsilon+a_{Q}+q_{h}\right)^{+}$. Just as before Theorem 6.2, we have $\tau\left(\varepsilon+a_{Q}\right) \in\langle h\rangle$, and this holds for all $h \in \mathcal{H}$.

Proposition 6.13. Suppose $Q^{\text {sat }}$ is simplicial (modulo units). Then there exists $a \in Q$ such that $a+\mathcal{E} \subset Q$.

Proof. The hypothesis on $Q$ means precisely that for each $i=1, \ldots, r$, the image $\tau(Q)$ contains an element $h^{i} \in \mathcal{H}$ in its Hilbert basis whose unique nonzero coordinate is $h_{i}^{i}>0$. Observe that $\left\langle h^{i}\right\rangle=\left\{\zeta \in \mathbb{Z}^{r} \mid \zeta_{i}>-h_{i}^{i}\right\}$ is a half-space by definition. Setting $\mathbf{h}=\left(h_{1}^{1}, \ldots, h_{r}^{r}\right) \in \mathbb{Z}^{r}$, we find that $\mathbf{h}+\bigcap_{h \in \mathcal{H}}\langle h\rangle \subseteq \mathbf{h}+\bigcap_{i=1}^{r}\left\langle h^{i}\right\rangle \subseteq \mathbb{N}^{r}$. By Corollary 6.12 we may take $a=a_{Q}+\widetilde{h}$ for any $\widetilde{h} \in Q$ satisfying $\tau(\widetilde{h})=\mathbf{h}$. 


\section{Infinite-dimensional socles.}

In this section we prove our principal result concerning semigroup rings, Theorem 7.1, by combining Theorem 5.8 with its converse, namely that if $Q^{\text {sat }}$ is not simplicial then one can always find an ideal $I$ for which local cohomology is not well-behaved. We avoid dealing with nontrivial units here, since they add nothing to the content, but obscure the statement.

Theorem 7.1. Let $Q$ be an affine semigroup of dimension $d$ with trivial unit group (but not necessarily saturated). The following are equivalent:

1. The saturation $Q^{\text {sat }}$ is simplicial.

2. For every $Q$-graded ideal $I$ and every finitely generated $Q$-graded $k[Q]$ module $M$, the Bass numbers of $H_{I}^{i}(M)$ are finite.

3. For every $Q$-graded prime $\mathfrak{p}$ of dimension $2, H_{\mathfrak{p}}^{d-1}\left(\omega_{k\left[Q^{\text {sat }}\right]}\right)$ has finitely generated socle.

This theorem provides a proof and generalization of Example 4.10. The key to our argument is Yanagawa's computation of the local cohomology of the canonical module $\omega_{k[Q]}$ over a normal semigroup ring $k[Q]$ [Yan01]. To state it, let $\tau_{1}, \ldots, \tau_{r}$ be linear functionals which vanish on the facets $F_{1}, \ldots, F_{r}$ of $Q$ and take nonnegative integer values on $Q$, as in the previous sections. For the sake of simplicity we assume that $Q$ has no nonzero units. Choose a hyperplane $H$ transverse to the real cone $\mathbb{R}_{+} Q$ generated by $Q$, so that $\bar{Q}=\left(\mathbb{R}_{+} Q\right) \cap H$ is a polytope of dimension $d-1=\operatorname{dim}(k[Q])-1$ whose faces (including the empty face $\varnothing$ ) correspond to the primes of $k[Q]$.

Definition 7.2. Let $F \in Q$ correspond to $\bar{F} \in \bar{Q}$ (so $\mathbf{0} \in Q$ corresponds to $\varnothing \in \bar{Q}$, for example). Define the polyhedral cell subcomplex

$$
\bar{F}(\alpha)=\left\{\overline{F^{\prime}} \in \bar{F} \mid\left(\alpha+\mathbb{R}_{+} Q\right) \cap F^{\prime}=\varnothing\right\}
$$

of $\bar{F}$ for any face $\bar{F} \subseteq \bar{Q}$ and $\alpha \in Q^{\text {gp }}$.

Theorem 7.3 ([Yan01, Theorem 6.1]). Let $Q$ be saturated and $\mathfrak{p}$ be a graded prime of $k[Q]$, corresponding to a face $\bar{F}$ of $\bar{Q}$. Then $H_{\mathfrak{p}}^{d-i}\left(\omega_{k[Q]}\right)_{\alpha} \cong$ $\widetilde{H}_{i-1}(\bar{F}, \bar{F}(\alpha))$ for all $\alpha \in Q^{\mathrm{gp}}$. $\bar{Q}$.

We will apply this when $\mathfrak{p}$ has dimension 2 ; that is, when $\bar{F}$ is an edge of

Proposition 7.4. If $\mathfrak{p}$ corresponds to the edge $\bar{F}$ and $Q$ is saturated, then:

1. $H_{\mathfrak{p}}^{d-1}\left(\omega_{k[Q]}\right)_{\alpha}=0$ if $\tau_{i}(\alpha)>0$ for some $i$ such that $\bar{F}_{i} \cap \bar{F} \neq \varnothing$.

2. $H_{\mathfrak{p}}^{d-1}\left(\omega_{k[Q]}\right)_{\alpha}=0$ if $\tau_{i}(\alpha) \leq 0$ for all $i$ such that $\bar{F}_{i} \cap \bar{F}=\varnothing$.

3. $H_{\mathfrak{p}}^{d-1}\left(\omega_{k[Q]}\right)_{\alpha}=k$ if neither of the above conditions holds.

Proof. Suppose the first condition holds. If $\bar{F}_{i}$ contains $\bar{F}$, then $\alpha+\mathbb{R}_{+} Q$ misses $F$ entirely, so $\bar{F}(\alpha)=\bar{F}$, and the zeroth relative homology is zero. 
Otherwise, $\bar{F}_{i} \cap \bar{F}$ is a vertex of the edge $\bar{F}$, and $\bar{F}(\alpha)$ contains at least that vertex. Thus the relative homology is again zero.

If the second condition holds, but the first does not, then $\tau_{i}(\alpha) \leq 0$ for all $i$. This implies $\bar{F}(\alpha)$ is the void complex - not even $\varnothing \in \bar{F}(\alpha)$, so the zeroth relative homology is still zero.

In the third case, $\bar{F}(\alpha)$ consists of just the empty face $\varnothing$, and the zeroth relative homology is the number of connected components of $\bar{F}$.

Corollary 7.5. Let $\bar{F}$ be an edge of $\bar{Q}$ such that there exists a facet $\bar{F}_{j}$ of $\bar{Q}$ with $\bar{F}_{j} \cap \bar{F}=\varnothing$. Let $\mathfrak{p}$ be the prime of $k[Q]$ corresponding to $\bar{F}$. Then if $Q$ is saturated, $H_{\mathfrak{p}}^{d-1}\left(\omega_{k[Q]}\right)$ has an infinite-dimensional socle.

Proof. Every nonzero element $x \in H_{\mathfrak{p}}^{d-1}\left(\omega_{k[Q]}\right)$ is annihilated by some power of the maximal ideal of $k[Q]$. To see this, suppose $x$ is homogeneous of degree $\alpha$, and assume that for some $\beta \in Q$, we had $\mathbf{x}^{n \beta} x \neq 0$ for all $n \in \mathbb{N}$. Then, by Proposition 7.4, $\tau_{i}(n \beta+\alpha) \leq 0$ for all $n$ and all $i$ such that $\bar{F}_{i} \cap \bar{F} \neq \varnothing$. Thus $\tau_{i}(\beta)=0$ for all such $i$, so $\beta \in \bar{F}_{i}$ for all such $i$. But the intersection of all such $\bar{F}_{i}$ is empty, so $\beta=\mathbf{0}$.

If $\bar{F}_{j} \cap \bar{F}=\varnothing$, then choose $\alpha \in Q^{\mathrm{gp}}$ such that $\tau_{j}(\alpha)>0$ and $\tau_{i}(\alpha) \leq 0$ for $i \neq j$. Proposition 7.4 implies that $H_{\mathfrak{p}}^{d-1}\left(\omega_{k[Q]}\right)_{\alpha}$ is nonzero and killed by some power of the maximal ideal, so $H_{\mathfrak{p}}^{d-1}\left(\omega_{k[Q]}\right)$ has nontrivial socle. Suppose its socle were finite-dimensional. Then there would exist $\beta \in Q^{\mathrm{gp}}$ such that $\tau_{j}(\beta)$ is maximal among the socle degrees in $Q^{\mathrm{gp}}$. But since $\tau_{j}(\alpha)>0$, we have $\tau_{j}(\beta)>0$, so $\tau_{j}(2 \beta)>\tau_{j}(\beta)$. Moreover the local cohomology is nontrivial in degree $2 \beta$. Taking a nonzero element of $H_{\mathfrak{p}}^{d-1}\left(\omega_{k[Q]}\right)_{2 \beta}$ and multiplying it by a sufficiently large power of the maximal ideal then yields a socle element in a degree $\gamma$ with $\tau_{j}(\gamma)>\tau_{j}(\beta)$, which is a contradiction.

Proof of Theorem 7.1. $1 \Rightarrow 2$ is Theorem 5.8, and $2 \Rightarrow 3$ is because $\omega_{k\left[Q^{\text {sat }}\right]}$ is finitely generated over $k[Q]$. For $3 \Rightarrow 1$, the unsaturated case follows from the saturated case. Indeed, any $Q^{\text {sat }}$-graded ideal $I \subset k\left[Q^{\text {sat }}\right]$ is generated up to radical by elements $\mathbf{y}=\left(y_{1}, \ldots, y_{s}\right)$ in $k[Q]$ (high powers of any homogeneous generating set for $I$ will do). If $M$ is any $k\left[Q^{\text {sat }}\right]$-module, the cohomology of the Cech complex $C^{\bullet}(\mathbf{y} ; M)$ on these generators is therefore a module over both $k\left[Q^{\text {sat }}\right]$ and $k[Q]$. As such, it is simultaneously the local cohomology of $M$ over $k\left[Q^{\text {sat }}\right]$ with support on $I \subset k\left[Q^{\text {sat }}\right]$ and over $k[Q]$ with support on $I \cap k[Q]$. Furthermore, any socle element of a $k\left[Q^{\text {sat }}\right]$-module is also a socle element over $k[Q]$, since the maximal ideal of $k[Q]$ is contained in the maximal ideal of $k\left[Q^{\text {sat }}\right]$.

Thus by Corollary 7.5, it suffices to produce, for any polytope $\bar{Q} \neq$ simplex, an edge $\bar{F}$ of $\bar{Q}$ that misses some facet. Equivalently, it suffices to show that if $\bar{Q}$ is a polytope in which every edge meets every facet then $\bar{Q}$ is a simplex. Let $\bar{F} \in \bar{Q}$ be a facet, and $\tau$ a linear functional supporting $\bar{F}$, nonnegative on $\bar{Q}$. Suppose $\tau$ takes a minimal nonzero value at a vertex 
$v \notin \bar{F}$. If more than one vertex of $\bar{Q}$ lies off of $\bar{F}$, there is an edge (necessarily missing $\bar{F}$ ) connecting $v$ to some vertex at which $\tau>0$. Thus, if every edge meets every facet, there can be only one vertex of $\bar{Q}$ lying off of each facet, and $\bar{Q}$ must be a simplex.

\section{Open problems.}

It has been seen above that affine semigroup rings provide a wealth of examples and counterexamples to general questions about local cohomology in singular varieties. In particular, they shed some light on some of the general questions posed by Huneke on local cohomology [Hun92]:

1. When is $H_{I}^{i}(M)$ zero?

2. When is $H_{I}^{i}(M)$ finitely generated?

3. When is $H_{I}^{i}(M)$ artinian?

4. When is the number of associated primes of $H_{I}^{i}(M)$ finite?

Although the answer to the fourth is trivially "always" in the cases discussed in this paper, the above examples provide clues as to how to refine the first three, given a grading.

Section 4 provides a possibility for answering Question 1: Relate the vanishing of local cohomology in a given cohomological degree to the vanishing of Ext modules in that cohomological degree and lower. Theorem 4.7 establishes this link for graded modules over semigroup rings; we believe that such a connection exists in significantly more generality, but we are unaware of how to relate infinitely generated modules to finitely generated ones without resorting to a grading. The key concept is that of a certain kind of "constancy", provided here by the Cech hull. This type of constancy is reminiscent of the characteristic 0 regular local case, in which the modules in question are treated as $D$-modules [Lyu93]. Perhaps the right generalization of $D$-module to the singular setting will provide the appropriate notion of constancy to bridge finitely generated Ext modules and local cohomology.

A partial answer to Question 2 in the general (local, ungraded) case concerns numerical criteria on the heights of primes and cohomological degrees involved [Hun92]. In the semigroup-graded case, finite generation can be viewed as a convex-geometric problem, dealing with $Q$-graded degrees in which the summands in a minimal injective resolution are nonzero. We expect in the $Q$-graded case for these considerations to yield geometric and combinatorial criteria in addition to the general numerical criteria. For the canonical module of a normal semigroup ring, for instance, local cohomology at a graded prime ideal $\mathfrak{p}$ of $R$ is finitely generated if and only if it vanishes, since Proposition 4.9 expresses such cohomology in terms of derived functors of $\check{C}$, which are never finitely generated if they are nonzero, or in terms of $\check{C} \omega_{R / \mathfrak{p}}$, which is also never finitely generated.

As pointed out by Huneke [Hun92], Question 3 has two parts, namely: 
3a. When is the maximal ideal the only associated prime of $H_{I}^{i}(M)$ ?

$3 \mathrm{~b}$. When are the Bass numbers of $H_{I}^{i}(M)$ finite?

Both $3 \mathrm{a}$ and $3 \mathrm{~b}$ should have concrete combinatorial answers in the semigroup case, at least when $M$ is a canonical module. In fact, we expect the essential set to play a pivotal role in answering these and the following refinement of 3a: For which cohomological degrees $i$ and graded ideals $I$ is a given prime of $k[Q]$ associated to $H_{I}^{i}\left(\omega_{k[Q]}\right)$ ?

As for Question 3b, it seems to be connected with the kinds of singularities which appear in the normalization of the ring $R$. Whether this holds in more generality than simply for semigroup rings is an interesting question. For instance, one can try classifying the singularities of a ring $R$ or ideals $I$ for which the modules $H_{I}^{i}(M)$ can have infinite Bass numbers (or, for that matter, which primes can appear with infinite Bass number). Even in the case of a semigroup ring, we do not have satisfactory answers to these last questions.

Finally, is there a global version of the Čech hull that works for toric varieties, and if so, what is its relation to the Čech hull over the Cox homogeneous coordinate ring [Cox95]? More generally, for varieties with a torus action, can a global Čech hull give information about cohomology with support on subvarieties fixed pointwise by subgroups of the acting torus? In the toric case, properties of a global Čech hull will be governed by the group of Weil divisors modulo Cartier divisors, introduced by Thompson to control resolutions of singularities [Tho01].

Acknowledgements. The authors wish to thank Bernd Sturmfels and Kohji Yanagawa for their useful suggestions. Both authors were partially supported by the National Science Foundation, and the second was partially supported by the Alfred P. Sloan Foundation.

\section{References}

[BS98] D. Bayer and B. Sturmfels, Cellular resolutions of monomial modules, J. Reine Angew. Math., 502 (1998), 123-140, MR 99g:13018, Zbl 0909.13011.

[BH93] W. Bruns and J. Herzog, Cohen-Macaulay Rings, Cambridge Studies in Advanced Mathematics, 39, Cambridge University Press, Cambridge, 1993, MR 95h:13020, Zbl 0788.13005.

[Cox95] D. Cox, The homogeneous coordinate ring of a toric variety, J. Algebraic Geom., 4 (1995), 17-50, MR 95i:14046, Zbl 0846.14032.

[ER98] J.A. Eagon and V. Reiner, Resolutions of Stanley-Reisner rings and Alexander duality, J. Pure Appl. Algebra, 130(3) (1998), 265-275, MR 99h:13017, Zbl 0941.13016.

[EMS00] D. Eisenbud, M. Mustaţă and M. Stillman, Cohomology on toric varieties and local cohomology with monomial supports, J. Symbolic Comput., 29(4-5) (2000), 
583-600, Symbolic computation in algebra, analysis, and geometry (Berkeley, CA, 1998), MR 2002a:14059.

[GW78] S. Goto and K. Watanabe, On graded rings, II ( $\mathbb{Z}^{n}$-graded rings), Tokyo J. Math., 1(2) (1978), 237-261, MR 81m:13022, Zbl 0406.13007.

[Har70] R. Hartshorne, Affine duality and cofiniteness, Invent. Math., 9 (1970), 145-164, MR 41 \#1750, Zbl 0196.24301.

[Hun92] C. Huneke, Problems on local cohomology, in 'Free Resolutions in Commutative Algebra and Algebraic Geometry' (Sundance, UT, 1990), Jones and Bartlett, Boston, MA, 1992, 93-108, MR 93f:13010, Zbl 0782.13015.

[HS93] C. Huneke and R. Sharp, Bass numbers of local cohomology modules, Trans. Amer. Math Soc., 339 (1993), 765-779, MR 93m:13008, Zbl 0785.13005.

[Ish88] M.-N. Ishida, The local cohomology groups of an affine semigroup ring, in 'Algebraic Geometry and Commutative Algebra in Honor of Masayaoshi Nagata', Vol. I, Kinokuniya, Tokyo, 1988, 141-153, MR 90a:13029, Zbl 0687.14002.

[Lyu93] G. Lyubeznik, Finiteness properties of local cohomology modules (an application of D-modules to commutative algebra), Invent. Math., 113(1) (1993), 41-55, MR 94e:13032, Zbl 0795.13004.

[Mil98] E. Miller, Alexander duality for monomial ideals and their resolutions. Preprint math.AG/9812095, 1998.

[Mil00] - The Alexander duality functors and local duality with monomial support, J. Algebra, 231(1) (2000), 180-234, MR 2001k:13028, Zbl 0968.13009.

[MP01] E. Miller and D. Perkinson, Eight lectures on monomial ideals, in 'COCOA VI: Proceedings of the International School', Villa Gualino, May-June, 1999 (A.V. Geramita, ed.), Queens Papers in Pure and Applied Mathematics, 120, Queen's Univ., Kingston, ON, 2001, 3-105.

[Mus00] M. Mustaţă, Local cohomology at monomial ideals, J. Symbolic Comput., 29(45) (2000), 709-720, MR 2001i:13020, Zbl 0966.13010.

[Ter99] N. Terai, Local cohomology modules with respect to monomial ideals. Preprint, 1999.

[Tho01] H. Thompson, On Toric Log Schemes, Ph.D. Thesis, University of California at Berkeley, 2002.

[Wei94] C.A. Weibel, An Introduction to Homological Algebra, 38, Cambridge University Press, Cambridge, 1994, MR 95f:18001, Zbl 0797.18001.

[Yan00] K. Yanagawa, Bass numbers of local cohomology modules with supports in monomial ideals, Math. Proc. Cambridge Phil. Soc., 131(1) (2001), 45-60, MR 2002c:13037.

[Yan01] _ Sheaves on finite posets and modules over normal semigroup rings, J. Pure Appl. Algebra, 161(3) (2001), 341-366, MR 2002k:13033, Zbl 0992.13005.

Received September 14, 2001 and revised November 8, 2001. 
E-mail address: dhelm@math.berkeley.edu

Mathematical Sciences Research Institute 1000 Centennial Drive

BeRKELEy CA 94720

E-mail address: emiller@msri.org 